\title{
The Scope of Pediatric and Adolescent Gynecological Problems in Kuwait
}

\author{
Latifah Hasan $^{\mathrm{b}}$ Michael F. Diejomaoh ${ }^{\mathrm{a}, \mathrm{b}}$ Jehad Al-Harmi ${ }^{\mathrm{a}, \mathrm{b}}$ Asiya T. Mohd ${ }^{\mathrm{a}}$ \\ ${ }^{\mathrm{a} D e p a r t m e n t}$ of Obstetrics and Gynecology, Faculty of Medicine, Kuwait University, and ${ }^{\mathrm{b}}$ Maternity Hospital, Kuwait
}

\section{Key Words}

Adolescents · Gynecological disorders · Kuwait

\begin{abstract}
Objective: To establish an insight into pediatric and adolescent gynecological disorders encountered in a tertiary care center in Kuwait. Subjects and Methods: A retrospective case cohort review of 89 case records of patients under the age of 19 years who were admitted to the Maternity Hospital, Kuwait, from October 2002 through September 2003 was undertaken. For a variety of reasons, 35 patients were excluded and the remaining 54 patients constituted the study population. The patients were divided into 5 groups $(A-E)$ : group $A=$ ovarian cysts, group $B=$ disorders of pregnancy, group $C=$ menstrual disorders, group $D=$ gynecological infections and group $E=$ miscellaneous. The clinical presentation and management of patients were analyzed. Results: The following spectrum of gynecological disorders was obtained: 13 ovarian cysts $(24.1 \%), 28$ disorders of pregnancy (51.8\%), 4 menstrual disorders (7.4\%), 2 infections (3.7\%) and 7 miscellaneous cases (13.9\%). Of the 54 patients, 36 (66.7\%) were Kuwaitis and the remaining 18 (33.3\%) were non-Kuwaitis. The mean age of the study population was 15.16 years, the median was 15.5 years, and the range was 8-19 years. The mean age of the patients with disorders of pregnancy was $17.52 \pm 1.16$ years, which is significantly higher than that of groups $A, C$ and $E$ ( $p=0.007,0.0001$ and 0.05 ).
\end{abstract}

The main presenting symptoms at admission were vaginal bleeding: 31 (57.4\%), abdominal pain: 17 (31.5\%), abdominal mass: 10 (18.5\%), and vomiting: 10 (18.5\%). Ultrasonography revealed that dermoid cysts were the most frequently encountered ovarian cysts. Patients were hospitalized for 1-7 days. Conclusion: Disorders of pregnancy and ovarian cysts were the most frequently encountered pediatric/adolescent gynecological disorders. It is recommended that there should be a dedicated specialized clinic to guarantee privacy and centralized discreet care for these patients.

Copyright $\odot 2010$ S. Karger AG, Basel

\section{Introduction}

Pediatric and adolescent gynecology has emerged as a subspecialty area in obstetrics and gynecology, attracting the interest of residents. This field, however, has not gained recognition as a subspecialty in Kuwait, although pediatric and adolescent gynecological disorders are also encountered there.

In 1988, the Department of Gynecology of Tampere University Hospital, Finland, established a gynecological outpatient clinic for girls $\leq 15$ years, with the sole purpose of assigning all supervision of gynecological problems of children and adolescents to a team of gynecologists [1]; this practice has been replicated in many centers in the developed world and has also been associated with

\section{KARGER \\ Fax +4161306 1234 \\ E-Mail karger@karger.ch}

www.karger.com
(C) 2010 S. Karger AG, Basel

1011-7571/10/0195-0384\$26.00/0

Accessible online at:

www.karger.com/mpp
Michael F. Diejomaoh

Department of Obstetrics and Gynecology, Faculty of Medicine

Kuwait University, PO Box 24923

Safat 13110 (Kuwait)

Tel. +965 2531 9601, Fax +965 533 8906, E-Mail michaeldiejo@ hotmail.com 
the establishment of residency programs [2]. In the UK, simultaneous milestones were achieved with the recognition of pediatric and adolescent gynecology as a specialty area and the establishment of the British Society for Pediatric and Adolescent Gynecology in the year 2000 [3]. The structure of this organization is remarkably similar to the North American Society of Pediatric and Adolescent Gynecology (NASPAG) and recognizes the need for a multidisciplinary approach to the management of the plethora of conditions that fall under the umbrella of pediatric and adolescent gynecology.

In the UK, children with growth and pubertal problems are seen by pediatric endocrinologists in special centers where gynecologists also participate in their care [3]. Pediatric or primary care physicians currently manage many pediatric and adolescent gynecological disorders in Kuwait, but the examination seldom includes a detailed pelvic examination. Most pediatric and adolescent gynecological problems (including pubertal, growth, endocrine and congenital problems) are currently managed at different locations in the primary health care centers, and pediatric and gynecological clinics in Kuwait. The number of patients requiring inpatient and emergency care has not been well established. It is therefore vital to ascertain the magnitude of pediatric and adolescent gynecological disorders in Kuwait.

Hence, we decided to design a pilot study to gain insight into pediatric and adolescent gynecological disorders encountered in a tertiary care center in Kuwait, to discover the pattern of presentation and principles of management.

\section{Subjects and Methods}

For the purpose of this study, all patients under 19 years of age were considered to be pediatric or adolescent patients. During the period from October 2002 to September 2003, the case records of all patients under the age of 19 years admitted to the Maternity Hospital, Kuwait, a tertiary referral hospital, were collected. Of the 89 pediatric and adolescent patients, 54 were studied and 35 excluded. The exclusion criteria were patients with pregnancy disorders later than 13 weeks of gestational age (24 patients) and patients who, though under 19 years, discharged themselves against medical advice before definitive management could be implemented (11 patients).

The data collected from medical records included age, nationality, presenting complaints, social biodata, educational background and employment status, menstrual history, past medical and surgical history and history of drug usage. The findings on physical examination, investigation performed and management undertaken for these patients were also established from the records.

Scope of Pediatric and Adolescent

Gynecological Problems
Table 1. Spectrum of gynecological disorders

\begin{tabular}{llrr}
\hline Group & Clinical diagnosis & $\mathrm{n}$ & \multicolumn{1}{c}{$\%$} \\
\hline A & ovarian cysts & 13 & 24.1 \\
B & disorders of pregnancy & 28 & 51.8 \\
C & menstrual disorders & 4 & 7.4 \\
D & infections & 2 & 3.7 \\
E & miscellaneous & 7 & 13.0 \\
\cline { 2 - 3 } Total & & 54 & 100.0 \\
\hline
\end{tabular}

For the purpose of this study, the pediatric and adolescent patients were divided into 5 groups according to the gynecological diagnosis: group $\mathrm{A}=$ patients presenting with ovarian masses, group $\mathrm{B}=$ disorders of pregnancy at less than 13 weeks of gestation, group $\mathrm{C}=$ disorders of menstruation, group $\mathrm{D}=$ genital tract infection, and group $\mathrm{E}=$ miscellaneous disorders. All patients were admitted to the hospital either through the emergency unit as emergency cases or through the outpatient department as elective cases. They were managed by the various units according to standard guidelines for clinical practice.

Apart from clinical examinations of these patients, ultrasonography was carried out in all the patients in groups A-C. Conservative treatment was the mainstay of treatment for many patients. Evacuation of the uterus was performed in 14 (25.9\%) patients with incomplete miscarriage and missed abortion, and laparoscopy was carried out in 3 patients with ectopic pregnancy and in 4 with ovarian cysts. Laparotomy and ovarian cystectomy were performed in another 7 (13\%) patients with complicated ovarian cysts. The vulval/vaginal lacerations were sutured and no blood transfusion was required. One pathologist evaluated all biopsies. Unfortunately, the histological result could be traced in only 5 cases: 3 of these were cases of mature cystic teratoma (dermoid cyst) and hemorrhagic cyst and granulosa cell tumor in 1 case each. Upon discharge from the hospital, patients were followed up in outpatient clinics. The outcomes for all patients were analyzed using the SPSS 16 software system. Statistical analysis was performed using the alternate Welch $t$ test. $p \leq 0.05$ was regarded as significant.

\section{Results}

The gynecological spectrum of disorders in the 54 patients is shown in table 1 . Pregnancy disorder was the most frequent, occurring in 28 (51.8\%) patients followed by ovarian cysts in 13 (24.1\%).

Clinical characteristics of the study population are outlined in table 2 . Thirty-six of the patients (66.7\%) were Kuwaitis and 18 (33.3\%) were non-Kuwaitis. The mean age of the study population was 15.16 years, with a median age of 15.5 years and a range of $8-19$ years. The mean age of the patients admitted with disorders of pregnancy (group B, $17.52 \pm 1.16$ years) was significantly higher 
Table 2. Clinical characteristics of the study population

\begin{tabular}{|c|c|c|c|c|c|}
\hline Mean age, years & $15.1 \pm 2.64$ & $17.52 \pm 1.16$ & $14.67 \pm 1.53$ & $15.5 \pm 3.54$ & $13.0 \pm 4.85$ \\
\hline Median (range), years & $16(10-18)$ & $18(14-19)$ & $15(13-16)$ & $15.5(13-18)$ & $12.0(8-14)$ \\
\hline Kuwaiti & $9(69.2 \%)$ & $17(60.7 \%)$ & $4(100 \%)$ & $2(100 \%)$ & $4(57.2 \%)$ \\
\hline Non-Kuwaiti & $4(30.8 \%)$ & $11(39.3 \%)$ & 0 & 0 & $3(42.8 \%)$ \\
\hline Arabs & $4(30.8 \%)$ & $11(39.3 \%)$ & 0 & 0 & $2(28.6 \%)$ \\
\hline Married & 0 & $28(100 \%)$ & $1(25 \%)$ & 0 & $3(42.8 \%)$ \\
\hline Single & $13(100 \%)$ & 0 & $3(75 \%)$ & $2(100 \%)$ & $4(57.2 \%)$ \\
\hline \multicolumn{6}{|l|}{ Occupation } \\
\hline Student & $13(100 \%)$ & $18(64.3 \%)$ & $4(100 \%)$ & $2(100 \%)$ & $7(100 \%)$ \\
\hline Housewife & 0 & $10(35.7 \%)$ & 0 & 0 & 0 \\
\hline
\end{tabular}

p values for mean ages: group B vs. A: 0.007 (significant); group B vs. C: 0.0001 (significant); group B vs. D: 0.579 ; group B vs. E: 0.05 (significant).

than that of patients in groups $\mathrm{A}, \mathrm{C}$ and $\mathrm{E}$ (B vs. $\mathrm{A}: \mathrm{p}=$ 0.007 ; $B$ vs. $C: p=0.0001 ; B$ vs. $E: p=0.05$ ). There was no significant difference between groups B and D (B vs. D, $p=0.579$ ). Whereas all patients in group $B$ were married, all patients in groups $A$ and $D$ were unmarried and the majority of the patients in groups $\mathrm{C}$ and $\mathrm{E}$ were also unmarried (table 2). The majority of the study population were students at high schools, higher colleges such as universities and technical colleges or teacher training institutions; only 10 (35.7\%) patients were housewives (table 2).

The most frequently reported symptoms of these patients at presentation for admission were vaginal bleeding: 31 patients (57.4\%), abdominal pain: 17 patients (31.5\%), abdominal mass: 10 patients (18.5\%), vomiting: 10 patients (18.5\%), and trauma to the vulva and vagina: 3 patients (5.6\%) (table 3). Vaginal bleeding was reported by patients presenting with miscarriage, menstrual disorders and trauma to the genital tract. Abdominal pain was the presenting symptom in patients with disorders of pregnancy (miscarriages) and ovarian cyst. Vomiting was also reported by patients with disorders of pregnancy and complicated ovarian cysts. Dilatation of the cervix was detected in 9 patients (16.7\%) admitted with incomplete miscarriage, and there were 10 patients (18.5\%) whose cervix remained undilated (threatened miscarriage, missed abortion and ectopic pregnancy). An abdominal mass was detected in 8 patients (14.8\%) who presented with ovarian cysts and evidence of tenderness was detected in $50 \%$ of those patients with abdominal mass (table 3). Vulval and vaginal lacerations were detected in 3 patients (5.6\%). These lacerations were due to injuries sustained from falls on sharp objects.

The variety of gynecological disorders diagnosed is outlined in table 4 . Disorders of pregnancy, mainly miscarriages (19 cases, $35.2 \%$ ), were the most frequently reported: 9 of these were admitted with incomplete miscarriage, 5 with missed abortion, 2 with threatened miscarriage and 3 with ectopic pregnancy.

Ovarian cysts were also frequently diagnosed. In 10 (76.9\%) of the 13 patients diagnosed with ovarian cysts, abdominal mass associated with pain and abdominal mass without pain were separately reported by the patients. Four (7.4\%) presented with torsion of ovarian cysts and $3(5.6 \%)$ with bilateral functional ovarian cysts. Ultrasonography demonstrated the various types of ovarian tumors, which included mature cystic teratomas (dermoid cysts and granulosa tumor), the variety of pregnancy disorders and the underlying ovarian and uterine changes involved in those patients with menstrual disorders. Histological reports that were available from 5 patients who were operated upon confirmed the ultrasonographic finding of mature cystic teratoma (dermoid cyst) and granulosa tumor. Menstrual disorders, menorrhagia and severe dysfunctional uterine bleeding (anovulatory bleeding) were reported in 2 patients each. Regarding the hospital stay, the patients in group A with ovarian cysts had the longest hospital stay (7 days); group E patients had the shortest stay (2 days); other patients were admitted for 3-5 days. 
Table 3. Clinical features at presentation

\begin{tabular}{lrr}
\hline \multirow{2}{*}{ Events } & \multicolumn{2}{l}{ Cases } \\
\cline { 2 - 3 } & $\mathrm{n}$ & $\%$ \\
\hline Presenting complaints & & \\
$\quad$ Vaginal bleeding & 31 & 57.4 \\
Abdominal pain & 17 & 31.5 \\
Abdominal mass & 10 & 18.5 \\
Vomiting & 10 & 18.5 \\
Trauma to vulva & 3 & 5.6 \\
Nausea & 2 & 3.7 \\
Ambiguous genitalia & 1 & 1.9 \\
Vaginal discharge & 2 & 3.7 \\
Physical signs & & \\
Dilated cervical os & 9 & 16.7 \\
Undilated cervical os & 10 & 18.5 \\
Nontender abdominal mass & 4 & 7.4 \\
Abdominal mass & 8 & 14.8 \\
Tender abdominal mass & 4 & 7.4 \\
Vulval/vaginal laceration & 3 & 5.6 \\
Tender adnexal mass & 3 & 5.6 \\
Bilateral ovarian cysts & 2 & 3.7 \\
Ovarian hyperstimulation & 1 & 1.9 \\
Ambiguous genitalia & 1 & 1.9 \\
\hline
\end{tabular}

Some patients had more than one clinical feature.

Table 4. Pattern of gynecological disorders

\begin{tabular}{lrr}
\hline \multirow{2}{*}{ Disorder } & \multicolumn{2}{l}{ Cases } \\
\cline { 2 - 3 } & $\mathrm{n}$ & $\%$ \\
\hline Miscarriages $^{1}$ & 19 & 35.2 \\
Uncomplicated ovarian cysts $^{1}$ & 8 & 14.8 \\
Hyperemesis gravidarum & 5 & 9.3 \\
Torsion of ovarian cysts $^{1,2}$ & 4 & 7.4 \\
Functional ovarian cysts & 3 & 5.6 \\
Ectopic pregnancy $_{\text {Trauma to genital tract }}$ & 3 & 5.6 \\
Menorrhagia $^{\text {Dysfunctional uterine bleeding }}$ & 3 & 5.6 \\
Labial infections $^{\text {Partial molar pregnancy }}$ & 2 & 3.7 \\
Cryptomenorrhea $^{1}$ & 2 & 3.7 \\
Primary amenorrhea $^{1}$ & 1 & 3.7 \\
Ambiguous genitalia & 1 & 1.9 \\
Primary infertility $^{1}$ & 1 & 1.9 \\
& 1 & 1.9 \\
\hline
\end{tabular}

\footnotetext{
${ }^{1}$ There was a combination of diagnoses in some cases.
}

2 Includes dermoid cysts and ovarian hyperstimulation.

\section{Discussion}

This study described the pattern of gynecological disorders in pediatric and adolescent patients seen at the Maternity Hospital Kuwait, a tertiary care referral center in Kuwait. The most frequent gynecological problems encountered were pregnancy disorders, ovarian cysts, menstrual disorders and infections. Other gynecological problems such as endocrinological disorders, sexual abuse and sexually transmitted diseases, which were common adolescent gynecological problems, reported in other studies [1], were not found among our patient population. The percentage of Kuwaitis and non-Kuwaitis reported in the study $(66.7 / 33.3 \%)$ is similar to the distribution of $\mathrm{Ku}$ waitis and non-Kuwaitis in our clinic population.

The pregnancy disorder most frequently encountered in this study was miscarriage (abortion), similar to the findings in a previous study [1]. It should be noted that whereas the abortions reported in this study [1] were all induced, the miscarriages reported in our study were spontaneous miscarriages in married adolescents who were anxious to retain their pregnancies. Induced abortions are a very rare phenomenon in our practice in $\mathrm{Ku}$ wait; such induced abortions will usually be legal, medically indicated and approved by specific medical boards designated to deliberate on such requests. This explains the findings that vaginal bleeding and abdominal pain, complaints commonly reported by patients with miscarriages, were the most common presenting complaints in patients with pregnancy disorders.

Ovarian masses are uncommon in children, accounting for only 1-1.5\% of all childhood malignancies [4], and are always referred to tertiary centers for further management, most probably because of the need to exclude malignancy and offer appropriate definitive treatment and surgery. Approximately $67 \%$ of pediatric ovarian masses are germ cell tumors and $10-17 \%$ of these are epithelial tumors [4-7]. In our study, ovarian cysts were the second most common gynecological problem in adolescents. The most common presenting sign was abdominal mass (76.9\%), which confirmed a previous report of 70\% [4] in whom the presenting sign for ovarian masses was abdominal pain and the clinical examination revealed an abdominal mass in all of them. Less commonly reported symptoms were nausea and vomiting, observed in patients with torsion of the ovarian mass [4], which was also observed in our study.

Diagnosis can be achieved by ultrasonography [1] as in our study where it demonstrated various types of ovarian tumors. The treatment for obviously benign ovarian tu- 
mors consisted of cystectomy or ipsilateral oophorectomy or salpingo-oopherectomy. Biopsy of the other ovary was considered unnecessary if the ovary was patently normal $[4,7]$; this modality of treatment was applied to all patients in our study who presented with ovarian masses. Laparoscopic surgical treatment has become the main mode of treatment for ovarian cysts in this age group in Kuwait, although some investigators still perform laparotomy for suspected dermoid cysts of the ovary. All 3 adolescent patients presenting with ectopic pregnancy in the current study were treated laparoscopically. Laparoscopic surgery is performed for all patients admitted to the Maternity Hospital, Kuwait, with a diagnosis of ectopic pregnancy, except in some exceptional cases where a patient presents with vascular instability, when laparotomy is performed quickly. The histopathological finding of mature cystic teratoma in 5 of 11 patients is at variance with what has been reported in the literature [4-7]. The second most common histological diagnosis of granulosa cell tumor is also at variance with previous reports [4-8]. This may be explained by missing data in 6 out of the 11 patients for whom operations were performed.

Menstrual problems represent $50 \%$ of adolescent gynecological complaints. The most common cause of prolonged or heavy bleeding in this young age group is anovulatory bleeding, usually due to immaturity of the hypothalamic-pituitary-ovarian axis. Other infrequent causes of such abnormal vaginal bleeding are foreign bodies, infection, uterine masses or trauma [9]. This is different from the observation in our study, where the incidence reported for menstrual disorders was only $7.4 \%$. Our reported low incidence was for severe cases of menstrual disorders that required admission. Menstrual disorders, which represent $50 \%$ of adolescent gynecological problems [10], are managed in primary health care centers and it is unusual to refer these patients to specialized centers unless they present with severe anemia or suspected congenital hematological disorders [1]. Treatment of many cases of less severe menstrual disorders was offered at the various clinics (primary health and gynecological/pediatric) in Kuwait. Whereas $5.6 \%$ of our patients presented with trauma to the genital tract, a fall was the major cause of injury in some studies, accounting for $40-56 \%$ of cases, similar to the findings of Chaveepojnkamjorn et al. [11].

In our study, there were no reported cases of pelvic inflammatory disease or sexually transmitted diseases. This finding was quite different from observations of another study [12] in England and Wales, where the rate of sexually transmitted diseases per 100,000 population was highest in the adolescent age group and females bore the brunt of the epidemic. Important sequelae of sexually transmitted diseases include ectopic pregnancy, infertility, chronic pain and cervical cancer [12]. Cultural and religious practices in Kuwait do not allow premarital sexual activity and this partially explains the total absence of these disorders in our study population. Our adolescent patients do travel overseas to other developing or developed/western countries with more liberal sexual lifestyles for vacation and further studies; however, these adolescent girls travel with adult family members who supervise/monitor their activities. Another explanation for the lack of reporting pelvic inflammatory disease or sexually transmitted diseases in our study may be a failure of patients concerned to report to hospital for treatment (our study was based on inpatients only).

There were no reported cases of growth and pubertal disorders reported in our study because those patients were most probably referred to pediatric endocrine clinics or adult endocrinologists who treat pubertal and growth disorders in Kuwait. Cases of sexual abuse were not reported in our study for a variety of reasons: failure to report such incidents or difficulties encountered with the collection of data in a retrospective study. Although we have managed a few cases of sexual abuse in our hospital, many of these cases have been referred to forensic medicine clinics.

In order to be able to conduct a prospective study, we recommend dedicated pediatric and adolescent gynecological clinics. It is our hope that our pilot study will generate further studies in our region in this developing subspecialty area and will motivate specialists to undertake subspecialty training in pediatric and adolescent gynecology.

\section{Conclusion}

Disorders of pregnancy and ovarian cysts were the most frequently encountered pediatric/adolescent gynecological disorders. It is recommended that there should be a dedicated specialized clinic to guarantee privacy and centralized discreet care for pediatric and adolescent gynecological patients. 


\section{References}

$>1$ Piippo S, Lenko HL, Laippala PJ: Experiences of special gynecological services for children and adolescents: a descriptive study. Acta Paediatr 1998;87:805-808.

$>2$ Muram D, Simmons KJ: Pattern recognition in pediatric and adolescent gynecology. J Pediatr Adolesc Gynecol 2008;21:103-108.

$\checkmark 3$ Edmonds DK: Pediatric and adolescent gynecology - The UK experience. J Pediatr Adolesc Gynecol 2002;15:265-270.

4 Yazici M, Etensel B, Gursoy H, Erkos M: Mucinous cystadenoma: a rare abdominal mass in childhood. Eur J Pediatr Surg 2002;12: 330-332.
5 Brown MF, Hebra A, McGeehin K, Ross AJ 3rd: Ovarian masses in children: a review of 91 cases of malignant and benign masses. J Pediatr Surg 1993;28:930-933.

6 Freud E, Golinsky D, Steinberg RM, Blumenfeld A, Yaniv I, Zer M: Ovarian masses in children. Clin Pediatr (Phila) 1999;38: 573-577.

7 Helmrath MA, Shin CE, Warner BW: Ovarian cysts in the pediatric population. Semin Pediatr Surg 1998;7:19-28.

8 Salmio LM, Vettenrafa K, Mottonen M, Heikinheimo M: Ovarian granulosa cell tumors in childhood. Pediatr Hematol Oncol 2002;19:145-156.
9 Strickland JL, Wall JW: Abnormal uterine bleeding in adolescents. Obstet Gynecol Clin North Am 2003;30:321-335.

10 Sorinola O, Nippani KJ, Cox C: Rare cause of irregular bleeding in 12-year-old girl. J Obstet Gynaecol 2002;22:330-332.

11 Chaveepojnkamjorn W, Pichainarong N, Pooltawee S: Unintentional injuries among children aged 1-4 years at home. Southeast Asian J Trop Med Public Health 2002;33: 642-646.

12 Balen AH, Fleming CH, Robinson A: Health needs of adolescents in secondary gynecological care. Hum Fertil 2002;5:127-132. 